\title{
Energy Analysis During Biped Walking
}

Filipe M. Silva

\author{
Department of Mechanical Engineering \\ University of Aveiro \\ 3810 Aveiro, Portugal
}

\begin{abstract}
This paper describes the dynamic analysis of biped locomotion systems. A planar biped is modelled and studied focusing on the problem of energy efficiency. First, the motion of the system is characterised in terms of a set of locomotion variables, namely: step length, hip height, hip ripple, hip offset, foot clearance and link lengths. Afterwards, three cost functions related to energy are proposed: absolute power, power dispersion and power lost. The aim is to understand the influence of these locomotion variables on the energy flow. The performance measures are discussed and the results compared with those observed in nature.
\end{abstract}

\section{Introduction}

In the last years a growing field of research in biped locomotion culminated in the development of a variety of prototypes $[2,4,6,11]$. A retrospective analysis shows that the design methodologies led to the reproduction of structures, functions and principles found in nature. In spite of these accomplishments, we are still in a primitive stage in understanding the motor control principles and the sensory integration subjacent to human walking. These questions have motivated several researchers in the pursuit of more efficient walking robots. Vukobratovic et al. [10] have proposed models and mechanisms to explain biped locomotion. In another perspective, Raibert and his colleagues [7] built hopping and running legged robots in order to study the major issues with dynamic balance.

Experimental studies of human locomotion $[3,5]$ support the hypothesis that the choice of a gait pattern is influenced by energy considerations. In repetitive and skilled movements, the motion control programming may attempt to produce walking patterns which are energy efficient due to the physical benefits derived. Bearing this fact in mind, a planar biped is modelled in order to:

- Characterise the biped motion in terms of a set of locomotion variables.

- Search for the optimal locomotion variables that minimise the proposed cost functions.

- Establish the correlation among these locomotion variables and the energy performance.

\author{
J.A. Tenreiro Machado \\ Department of Electrical Engineering \\ Polytechnic Institute of Porto \\ Rua de S. Tomé, 4200 Porto, Portugal
}

The remainder of the paper is organised as follows. A short description of the biped model is given in section 2 . In section 3 we describe the algorithm used to plan the kinematic trajectories of the biped robot. In section 4 three energy performance measures are proposed and discussed mathematically. Based on these indices, several numerical results are presented in section 5 to illustrate the application of the proposed methods. Finally, in section 6 we outline the main conclusions and the perspectives towards future research.

\section{Biped Model}

Figure 1 shows the planar biped model with the notation used throughout this paper. The proposed model consists of seven links in order to approximate locomotion characteristics similar to those of the lower extremities of the human body (i.e., body, thigh, shank). In the present study, we assume that a complete walking cycle is divided in two phases:

- Single support phase in which one leg is in contact with the ground and the other leg swings forward.

- Exchange of support in which the legs trade role.

In the single support phase, the stance leg is in contact with the ground and carries the weight of the body, while the swing leg moves forward in preparation for the next step. At the exchange of support, the swing leg contacts the ground with zero velocity to smooth impulsive forces due to the impact phenomenon [12].

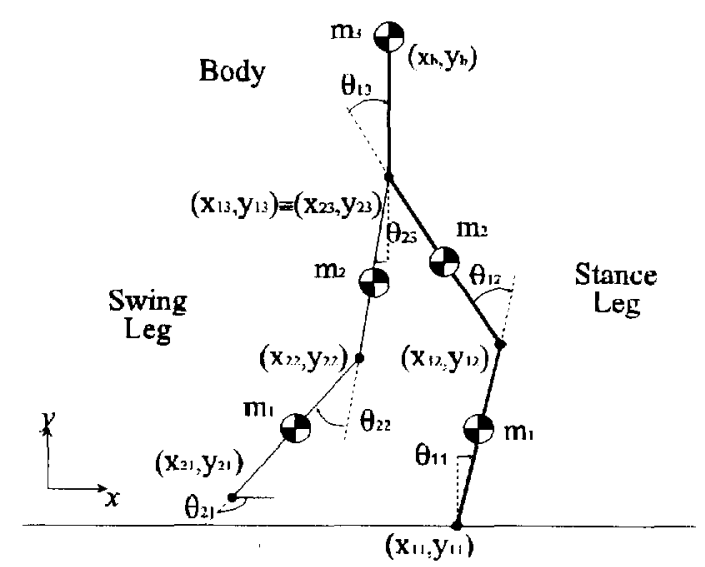

Figure 1: Planar biped model. 
The impact of the swing leg is assumed to be perfectly inelastic while ensuring that no slippage occurs. Using the Newton-Euler formulation, we derive the dynamic equations for the seven-link biped that describe the behaviour of a complete single step. The redundancies in the exchange of support are used to satisfy the torque continuity between the single-support and the doublesupport phases. At the time that the swing leg contacts the ground, an adequate reaction force is prescribed allowing a smooth transition of support. In this study, the ratio of the duration period of exchange of support and complete step is constant $(10 \%)$.

\section{Motion Planning and Evaluation}

In early work, the determination of the biped trajectories was made largely on the basis of experience and intuition (e.g., recording kinematic data from human locomotion) [1,9]. In this work, the motion planning is accomplished by prescribing the cartesian trajectories of the body and the lower extremities of the leg.

\subsection{Locomotion variables}

The motion of the biped system is characterised in terms of a set of variables. The step length $L_{S}$ is the distance travelled by the body in each step. The hip height $H_{H}$ is defined as the mean height of the hip along the walking cycle. The hip ripple $H_{R}$ is measured as the peak-to-peak oscillation magnitude at the hip. The hip offset $H_{O}$ measures the position of the hip in relation to the middle point between two consecutive contacts of the feet on the ground (i.e., $H_{O}=D_{1}-D_{2}$ ).

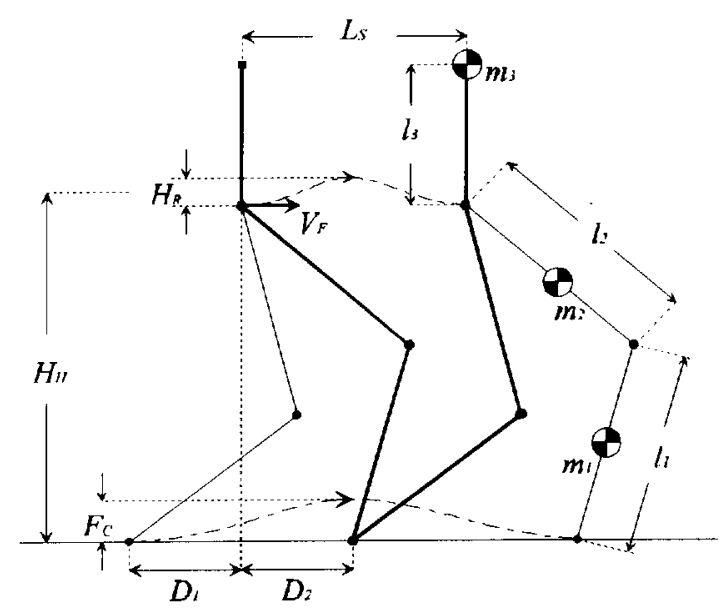

Figure 2: Locomotion variables.
The foot clearance $F_{C}$ represents the maximum elevation of the swing foot above the ground. Finally, we consider the link lengths and masses $l_{1}, l_{2}$ and $m_{1}, m_{2}$, respectively (Fig. 2).

\subsection{The Trajectory Generator}

The proposed algorithm accepts the hip and feet's cartesian trajectories as inputs and, by means of the inverse kinematics, generates the corresponding joint evolution. To improve the smoothness of the motion we impose two restrictions: the body maintains an erect posture and the body forward velocity $V_{F}$ is constant.

In dynamic walking, at each footfall, the system may suffer impacts and incurs on additional accelerations that influence the forward velocity. For this reason, we impose a set of conditions on the leg velocities so that the feet are placed on the ground while avoiding the impacts. We denote the moment of exchange of support as time $t_{1}$, and by $t_{1}^{-}$and $t_{1}^{+}$the instants just before and after the impact occurs, respectively. For a smooth exchange-ofsupport, we require that the angular velocities, before and after, to be identical, that is:

$$
\dot{\theta}_{2 i}\left(t_{1}^{-}\right)=\dot{\theta}_{1 i}\left(t_{1}^{+}\right)
$$

The locomotion parameters characterise completely any configuration in which both feet are on the ground. Nevertheless, between two such configurations there is an infinite number of possible trajectories. In order to simplify the problem, we consider that such motions are produced based on sinusoidal functions. The equation of the tip of the swing leg along the $x$-axis is computed by summing a linear function with a sinusoidal function. This is implemented using the function:

$$
x_{21}(t)=2 V_{F}\left[t-\frac{1}{2 \pi f} \sin (2 \pi f t)\right]
$$

where $f$ is the step frequency. Moreover, the vertical motion, that allows the foot to be lifted, has the form:

$$
y_{21}(t)=\frac{F_{C}}{2}[1-\cos (2 \pi f t)]
$$

The trajectory generator synchronises and coordinates the leg behaviour so that the swing limb arrives at the contact point when the upper body is properly centred with respect to the lower limbs. These trajectories are somewhat restrictive when compared with those of humans where we have ballistic-like motions. In this perspective, the sinusoidal trajectories constitute, merely, a first-order approach to more efficient movements. 


\subsection{Performance Evaluation}

After planning the joint trajectories, we calculate the inverse dynamics in order to map the kinematics into power consumption. The key measure of this analysis is the average mechanical power. It is computed assuming that power regeneration is not available by motors doing negative work, that is, by taking the absolute value of the power. At a given joint $j$ of the leg $i$, the mechanical power is the product of the motor torque and the angular velocity. The global index is obtained by averaging the mechanical absolute power delivered over a period $T$ :

$$
P_{m}=\frac{1}{T} \sum_{i, j} \int_{0}^{T}\left|\tau_{i j} \dot{q}_{i j}\right| d t
$$

Although minimizing energy appears to be an important consideration, it may occur an instantaneous near-infinite power demand. In such a case, the average value can be small while the peak is physically unrealizable. As an alternative index, the standard deviation measure is used to evaluate the dispersion around the mean absolute power:

$$
D_{m}=\sqrt{\frac{1}{T} \int_{0}^{T}\left(P_{i}-P_{m}\right)^{2} d t}
$$

where $P_{i}$ is the total instantaneous mechanical power.

For an actuated system, it should be also necessary to consider the energy lost in the electric motors. This index can be defined as:

$$
L_{e}=\frac{1}{T} \int_{0}^{T} \tau^{T} \tau d t
$$

\section{Simulation Results}

In this section, we describe the simulation results obtained using the different performance indices. At this stage, the relative performance indices by itself are not sufficient to address the issues of dynamic stability or control. The main purpose is to determine the implications of the locomotion variables on the energy flow and to compare the results with biological data. The simulations are carried out considering a total system mass and body height of $M=70 \mathrm{~kg}$ and $L=1.8 \mathrm{~m}$, respectively.

\begin{tabular}{c|cc}
\hline$i$ & $l_{i}(\mathrm{~m})$ & $m_{i}(\mathrm{~kg})$ \\
\hline 1 & 0.5 & 4.0 \\
2 & 0.5 & 7.5 \\
3 & 0.3 & 47 \\
\hline
\end{tabular}

Table I - The robot link lengths and masses.

\subsection{Step Length and Hip Height}

In a first case study we analyse (Fig. 3) the performance index $P_{m}$ with respect to the step length and the hip height. For convenience, the chart portion corresponding to hip heights lower than $0.5 \mathrm{~m}$ is not represented. One trajectory that undergoes smooth motion is the body flat trajectory in which the stance leg adjusts itself so that the hip maintains a constant height. The body of the robot is assumed to be moving horizontally with a constant forward velocity $V_{F}=1 \mathrm{~ms}^{-1}$. Furthermore, it is considered that the swing foot stays always on the ground. The system performance is particularly sensitive to the body mass above the hip. Therefore, the link length $v s$ mass values adopted in this study are based on anthropometric data [9] (Table I).

As shown in figure 3, to minimize the average mechanical power $P_{m}$ the hip height must be about $85 \%$ of the body height, with optimal step lengths in the range of 0.5-0.7 m. Moreover, an important degradation occurs for small step lengths. In figure 4 , we depict the results when evaluating the energy lost $L_{e}$. The performance surface presents a similar evolution, but $L_{e}$ is minimized for a slightly higher hip height. At the same time, these results agree with those observed in human locomotion when a subject is allowed to walk without the imposition of a pace frequency constraint.

The contour plot in figure 5 shows the dispersion measure $D_{m}$. The standard deviation allows the estimation of undesirable abrupt power transitions. As we can observe, the range of minimal dispersion corresponds to smaller hip heights, while the optimal step length is about $0.6 \mathrm{~m}$.

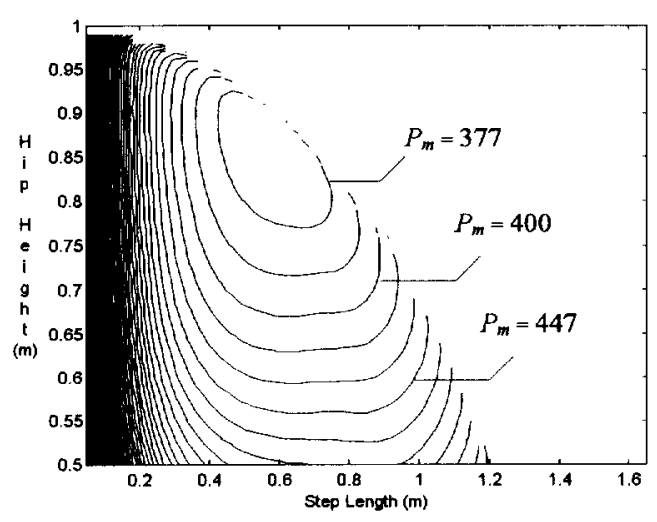

Figure 3: Mean absolute mechanical power $P_{m}$ vs $H_{H}$ and $L_{S}$. 


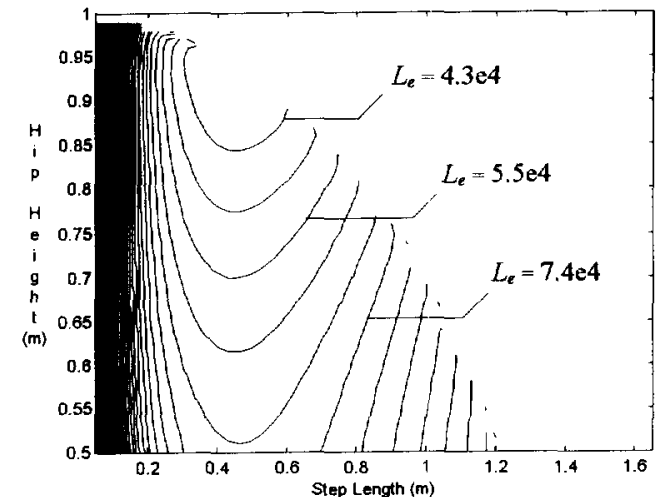

Figure 4: Mean power lost $L_{e} v s H_{H}$ and $L_{S}$.

In order to reproduce the role of each individual joint we compare their relative weights (Fig. 6(a-d)). At the point of minimum global power $P_{m}\left(L_{S}=0.55 \mathrm{~m}\right.$ and $H_{H}=0.87 \mathrm{~m}$ ) the stance ankle and the swing hip dominate the power requirements. Furthermore, the swing knee presents significant requirements for increasing hip heights. This means that driving totally the swing leg can be expensive. A possible alternative to be further investigated is to use the gravitational oscillation of the swing leg as a pendulum.

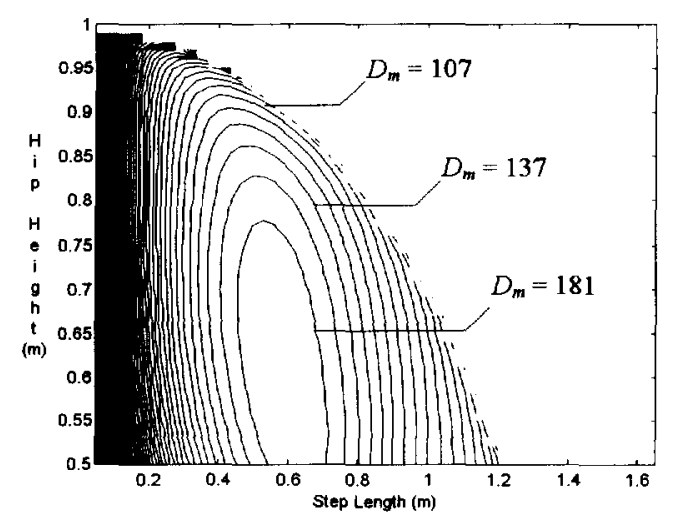

Figure 5: Mean power dispersion $D_{m}$ vs $H_{H}$ and $L_{S}$.

A smaller (higher) forward velocity diminishes (increases) the power requirements. In this case, the optimal step length decreases (increases) slightly, while the hip height tends to the maximum (minimum) value. The optimal locations of the pair $\left(L_{S}, H_{H}\right)$, for different values of forward velocity $V_{F}$, exhibit the evolution plotted in figure $6(e)$. At the same time, the corresponding optimal global power requirements increase with the forward velocity $V_{F}$ close fitting a cubic curve (Fig. 6(f)).

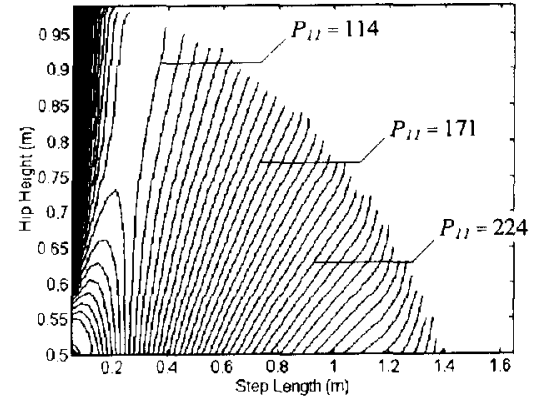

(a)

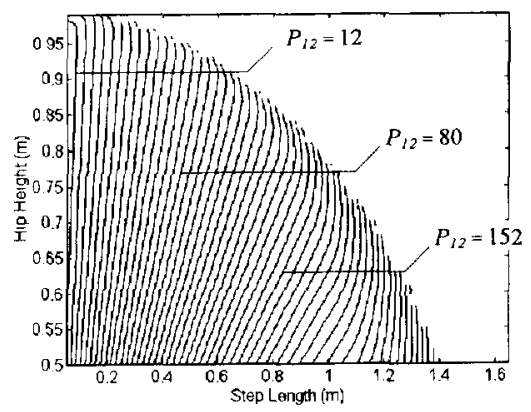

(b)

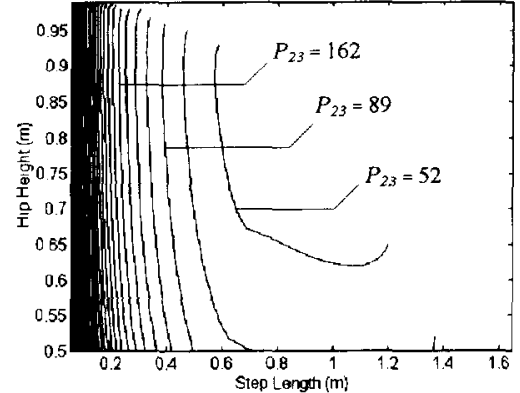

(c)

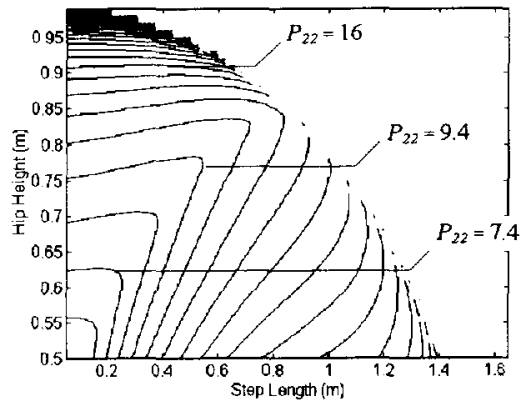

(d)

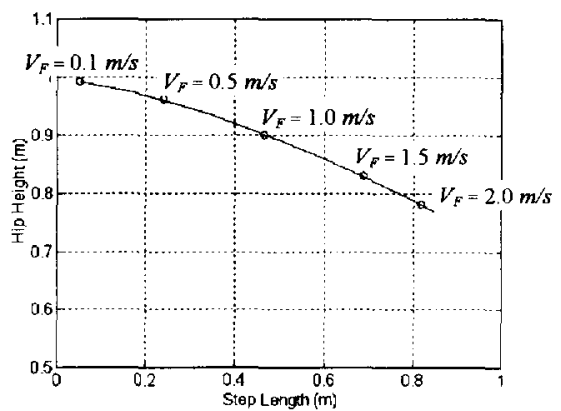

(e)

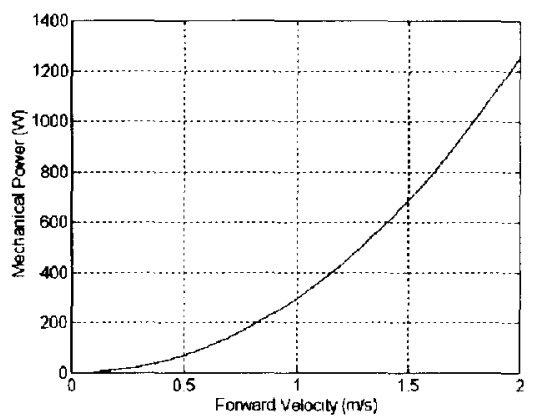

(f)

Figure 6: Contour plots of the absolute power $P_{m}$ at the individual joints: $(a)$ stance ankle, $(b)$ stance knee, (c) swing hip, (d) swing knee; (e) Optimal $\left(L_{S}, H_{H}\right)$ locus $v s$ forward velocity $V_{F},(f)$ Absolute power $v s$ forward velocity $V_{F}$. 


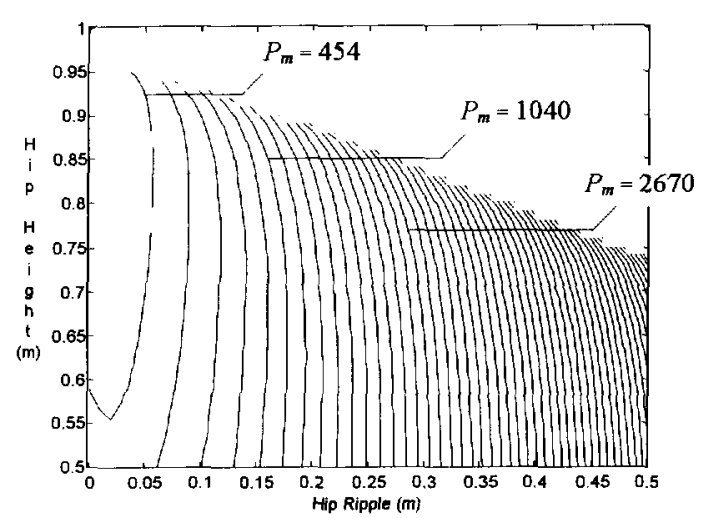

Figure 7: Mean absolute mechanical power $P_{m}$ vs $H_{H}$ and $H_{R}\left(L_{S}=0.4 \mathrm{~m}\right.$ and $\left.H_{O}=F_{C}=0 \mathrm{~m}\right)$.

\subsection{Hip Ripple}

In this sub-section, we consider a hip trajectory with a sinusoidal oscillation, while the foot of the swing leg slides over the ground surface during all the cycle. The contour plot in figure 7 suggests that a small adjustable oscillation at the hip may be advantageous. Furthermore, this value remains almost unchanged to hip height variations. A similar property can be observed in biological systems as well as in previous studies concerning the kinematic analysis of biped systems [8].

\subsection{Hip Offset}

The desired leg coordination is established by assuring that the swing leg arrives at the contact point when the upper body is properly centred with respect to both feet. However, the experiments carried out indicate the advantage of applying a positive offset to the hip.

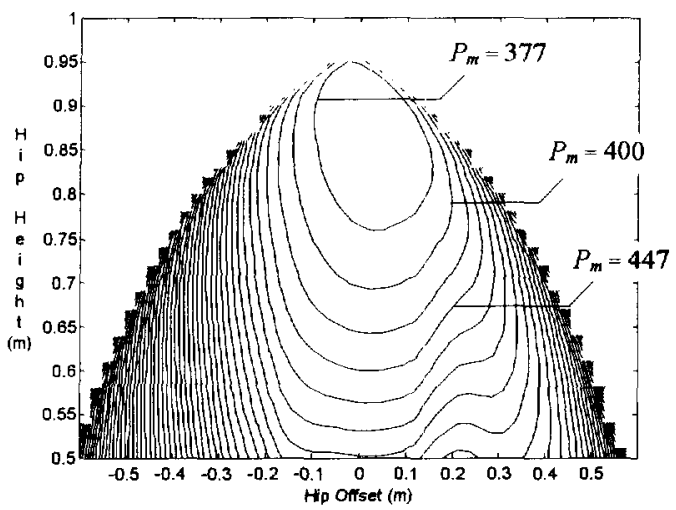

Figure 8: Mean absolute mechanical power $P_{m}$ vs $H_{H}$ and $H_{O}\left(L_{S}=0.4 \mathrm{~m}\right.$ and $\left.H_{R}=F_{C}=0 \mathrm{~m}\right)$.
Figure 8 represents the contour plot of the cost in terms of hip offset and hip height. Considering the above plot, two features can be pointed out:

- The optimal hip offset is always positive.

- The hip offset tends to zero as the hip height increases.

The stance and the swing legs present opposed behaviours suggesting a compromise in the final result. The benefits of a small hip offset results from the fact that, during walking, the legs move back and forth to provide, simultaneously, propel and balance actions.

\subsection{Foot Clearance}

Until now, all the experiences considered that the foot stays on the ground without any friction. Next, we analyse the situation in which the foot can be lifted off the ground. Figure 9 shows the influence of the foot clearance variable when using the absolute power as our performance index. In terms of the index $P_{m}$, the minimum foot clearance is the optimal one. Although less efficient from the $P_{m}$ perspective, we believe that the foot clearance is responsible for the system's robustness in uneven walking surfaces. We can observe this process in one-year-old infant's first steps knowing that walking will be learned (when avoiding any accidental contact)

The results obtained with the power lost index $L_{e}$ are shown in figure 10. It is possible to confirm that a zero foot clearance optimise the energy performance.

This means that a cost function based on energy may not be appropriated in this circumstances. As a result, a complementary performance index is currently under development to capture this phenomenon. At the same time, we expect that this issue will gain even more importance when incorporating other phenomena of the gait in our models.

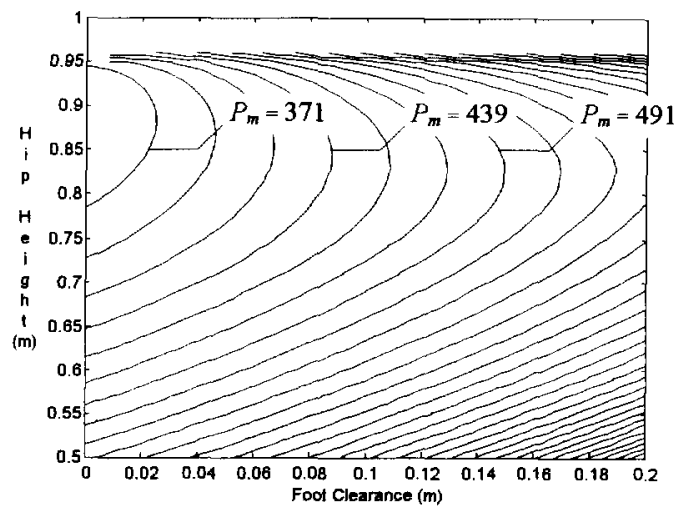

Figure 9: Mean absolute mechanical power $P_{m}$ vs $H_{H}$ and $F_{C}\left(L_{S}=0.4 \mathrm{~m}\right.$ and $\left.H_{R}=H_{O}=0 \mathrm{~m}\right)$ 


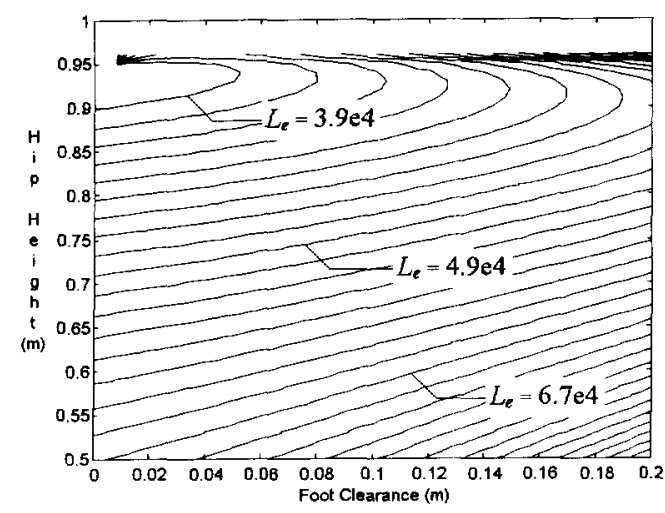

Figure 10: Mean power lost $L_{\mathrm{e}}$ vs $H_{H}$ and $F_{C}$ $\left(L_{S}=0.4 \mathrm{~m}\right.$ and $\left.H_{R}=H_{O}=0 \mathrm{~m}\right)$.

\subsection{Relative link lengths}

This sub-section investigates the role of the relative link lengths in the system's performance considering $l_{1}+l_{2}=1 \mathrm{~m}$. The minimum cost occurs for link lengths $l_{1}=0.65$ and $l_{2}=0.35 \mathrm{~m}$. The values of mean power are plotted against link length $l_{1}$ in figure 11 . Moreover, the results indicate that for $l_{1}$ in the range from 0.4 to $0.8 \mathrm{~m}$ the performance index remains almost constant.

\section{Conclusions}

In this paper, we have studied several aspects of biped locomotion. By implementing different motion patterns, we estimated how the robot reacts to a variety of locomotion variables: step length, hip height, hip ripple, hip offset, foot clearance and link lengths. The performance indices used provide a way to evaluate the system's behaviour during normal walking. Moreover, the simulation results could be used to gain insight into the implications of many design and motion planning parameters on the energy efficiency of a bipedal system.

Future work will address the refinement of our models to include other phenomena of the gait, such as lateral balance and zero ankle torque. At an higher level, it is essential to explore complementary performance measures (e.g., stability, obstacle avoidance) for the generation of efficient motion strategies.

\section{Acknowledgments}

The first author is supported by the Fundação para a Ciência e a Tecnologia under grant PRAXIS $\mathrm{XXI} / \mathrm{BD} / 9541 / 96$.

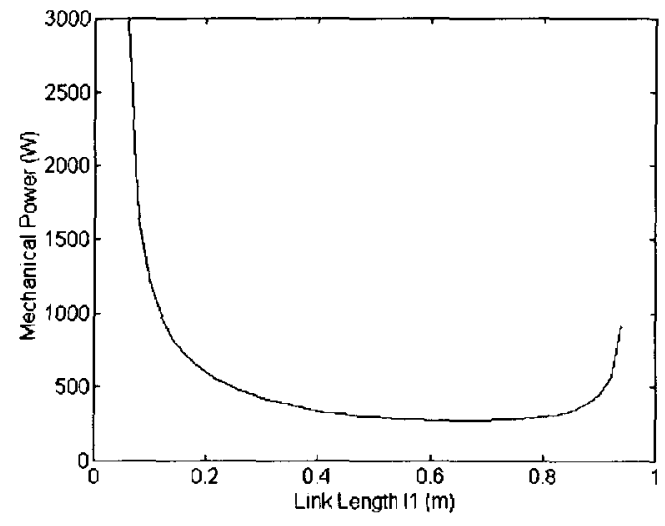

Figure 11: Performance index $P_{m} v s$ link length $l_{1}$.

\section{References}

[1] J. Basmajian and C. Luca, Muscles Alive: Their Functions Revealed by Electromyography, Williams \& Wilkins, 1978.

[2] J.A. Golden and Y.F. Zheng, "Gait Synthesis for the SD-2 Biped Robot to Climb Stairs", Int. Journal of Robotics and Automation, Vol. 5, n. 4, 1990.

[3] N.C. Heglund, C.R. Taylor, "Speed, Stride Frequency and Energy Cost per Stride - how do they change with body size and gait?", J. Exp. Biol., Vol. 138, pp. 301-318, 1988.

[4] K. Hirai, M. Hirose, Y. Haikawa and T. Takenaka, "The Development of Honda Humanoid Robot", Proc. IEEE Int. Conf. on Robotics and Automation, pp. 1321-1326, Leuven, Belgium, 1998.

[5] A. Hreljac and P.E. Martin, "The Relationship Between Smoothness and Economy During Walking", Biological Cybernetics, Vol. 69, pp. 213-218, 1993.

[6] S. Kajita, K. Tani, "Experimental Study of Biped Dynamic Walking", IEEE Control Systems, pp. 13-19, 1996.

[7] M.H. Raibert, Legged Robots that Balance, The MTT Press, 1986.

[8] F. Silva and J.A. Tenreiro Machado, "Kinematic Aspects of Robotic Biped Locomotion Systems", Proc. IEEE Int. Conf. on Intelligent Robots and Systems, IROS'97, Vol. 1, pp. 266-271, 8-13 Sept., Grenoble, France, 1997.

[9] D.A. Winter., Biomechanics and Motor Control of Human Movement, John Wiley \& Sons, 1990.

[10] M. Vukobratovic B. Botovac, D. Surla and D. Stokic, Biped Locomotion: Dynamics, Stability, Control and Applications, Springer-Verlag, 1990.

[11] J-I Yamaguchi, A. Takanishi and I. Kato, "Development of a Biped Walking Robot Adapting to a Horizontally Uneven Surface", Proc. Int. Conf. on Intelligent Robots and Systems, pp. 1156-1163, 1994.

[12] Y.F. Zheng and H. Hemami, "Impact Effects of Biped Contact with the Environment", IEEE Trans. Syst. Man Cyber., Vol. 14, n. 3, pp. 437-443, 1984. 